\title{
RESEARCH INTO VISUAL - MOTOR MONITORING BY OPERATORS OF MOBILE OBJECTS
}

\author{
Igor Petukhov* \\ Volga State University of Technology, Russian Federation \\ Lyudmila Steshina \\ Volga State University of Technology, Russian Federation \\ Pavel Kurasov \\ Volga State University of Technology, Russian Federation \\ Dmitriy Chernykh \\ Volga State University of Technology, Russian Federation \\ II'ya Tanryverdiev \\ Volga State University of Technology, Russian Federation
}

The paper presents a new model of the visual-motor monitoring which features two control loops - objective and subjective ones. This model allows to explain the phenomenon of cognitive dissonance that is experienced by operators in solving sophisticated problems. The results of experimental studies comprising the control of an object under conditions of the objective and subjective control are presented.

Keywords: Visual-motor monitoring; Oerator; Ergatic control systems; Response to a mobile object.

\section{INTRODUCTION}

The modern concept of reliable and safe ergatic control systems (ECS) is based on optimal distribution of functions between the man and the machine that provides accomplishment and improvement of each subsystems and balances their weaknesses.

The human-operator is remaining one of the most universal control systems and features a high capability to adaptation, training, an ability to make decisions under conditions of lacking information and a great shortage of time.

At the same time, the human is believed to be highly susceptible to the influence of external and internal disturbing factors, to be less reliable in comparison with a technical system, to have a lower calculating capacity and accuracy.

Nevertheless, from the point of view of functional systems, the reliability of the human and flexibility of its provision is extremely high even in comparison with technical systems of multiple-circuit reservation. The possibility to use lowly formalized associative enquiries to a memory, the capability to operate with significant blocks of fuzzy information, adaptable interface possibilities pro- vide a noticeable advantage of the human over a technical system in controlling the most complex ECS.

On the other side, sometimes the capability of the human to a fuzzy processing of lowly formalized information becomes a source of an additional "human factor". The literature review gives the description of a cognitive dissonance when the objective control data do not coincide with the human-operator's subjective perception of the control process. As a result, the human-operator making a decision has to choose, which data in his/her opinion are more confident and which data they would use. In its turn, this determines the control program and the outcome. It should be noted, that under condition of cognitive dissonance the operator habitually trusts his/her own subjective sensations, but not the means of an objective control.

It is just a cognitive dissonance that very often becomes a source of "a human factor" in the ECS as well as an explanation of illogical, at first sight, actions of the operator contradicting to standard instructions, objective instrument readings, etc. 
The founder of the cognitive dissonance theory is Leon Festinger. In his work [01] he introduces such notions as dissonance and relevance. Festinger refers relevance to the relation of a logic connection between two elements (cognitions) in any description, and dissonance to a degree of contradiction arising between two cognitions. Additionally, the term of consonance is introduced, i.e. lack of dissonance. It is important to understand that the individual under a dissonance condition is always trying to eliminate it by changing his/her behavior or knowledge of this phenomenon, or adding new cognitive elements to existing knowledge.

The background of cognitive dissonance occurrence and the further development of the cognitive dissonance theory are analyzed by Irem Metin and Selin Metin Camgoz [02].

E. Harmon-Jones [03] studied the cognitive dissonance theory considering three different dissonance phenomena in a human behavior which do not contradict the concept of its occurrence, they are self-consistency, self-affirmation and spreading of alternatives. The author proposed his own action-based model of dissonance that, in a way, allows to assess causes of its occurrence.

The issues of dissonance influence on decision-making processes were experimentally investigated by D. Dickinson, R. Oxoby [04]. The experiments showed that the dissonance once aroused affects making all further decisions while the scopes considered may be different.

Khodakov et al. [05] suggested describing the relevance and dissonance conditions using fuzzy logic and introduced specific terms and essential requirements to the usage of assessment meth- ods for contradictory knowledge. The authors also note that dissonance plays a stimulating role in the cognitive process, and understanding of dissonance occurrence and existence will facilitate formalization of the human cognitive activity aspects.

The phenomenon of cognitive dissonance can occur practically at any stage of an operator activity [06], but its influence on the decision-making process is different at each stage.

G.T.Beregovoy and V.A.Ponomarenko [07] in the context of test pilots training considered the influence of a pilot's psychological factors on decision-making in extreme situations. The authors believe that artificial introduction into a dissonance condition is an effective tool for development of operational thinking.

On the other side, the information on decisionmaking under cognitive dissonance conditions at all stages of an operator's activity can facilitate development of a theoretical base for designing modern intellectual tools for an operator's activity support which do not conflict with a user.

Thus, the research objective arises which is connected with investigation of an operator's activity in the ECS structure and assessment of subjective sensation influence on a decision-making mechanism in the control process including the conditions of cognitive dissonance that is a goal of this paper.

\section{DESKTOP ANALYSIS}

Nowadays mostly man-machine systems of control are usually related to the ECS class as Figure 1 shows.

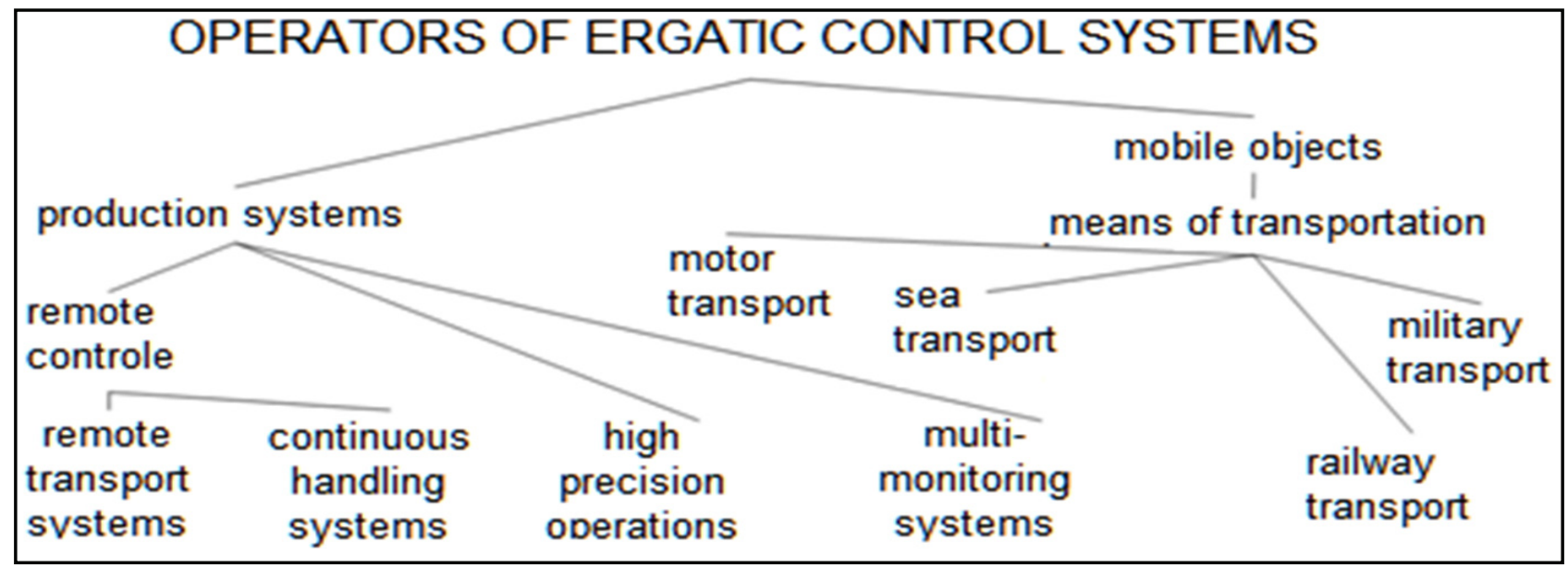

Figure 1. Classification of operators due to a controlled object 
The main features of such systems are social and psychological aspects of control inherent to a human-operator or a group of operators. specific, nevertheless, the following typical stages of an operator's activity can be determined practically for all operators, Figure 2.

Operators' functions for each ECS group is rather

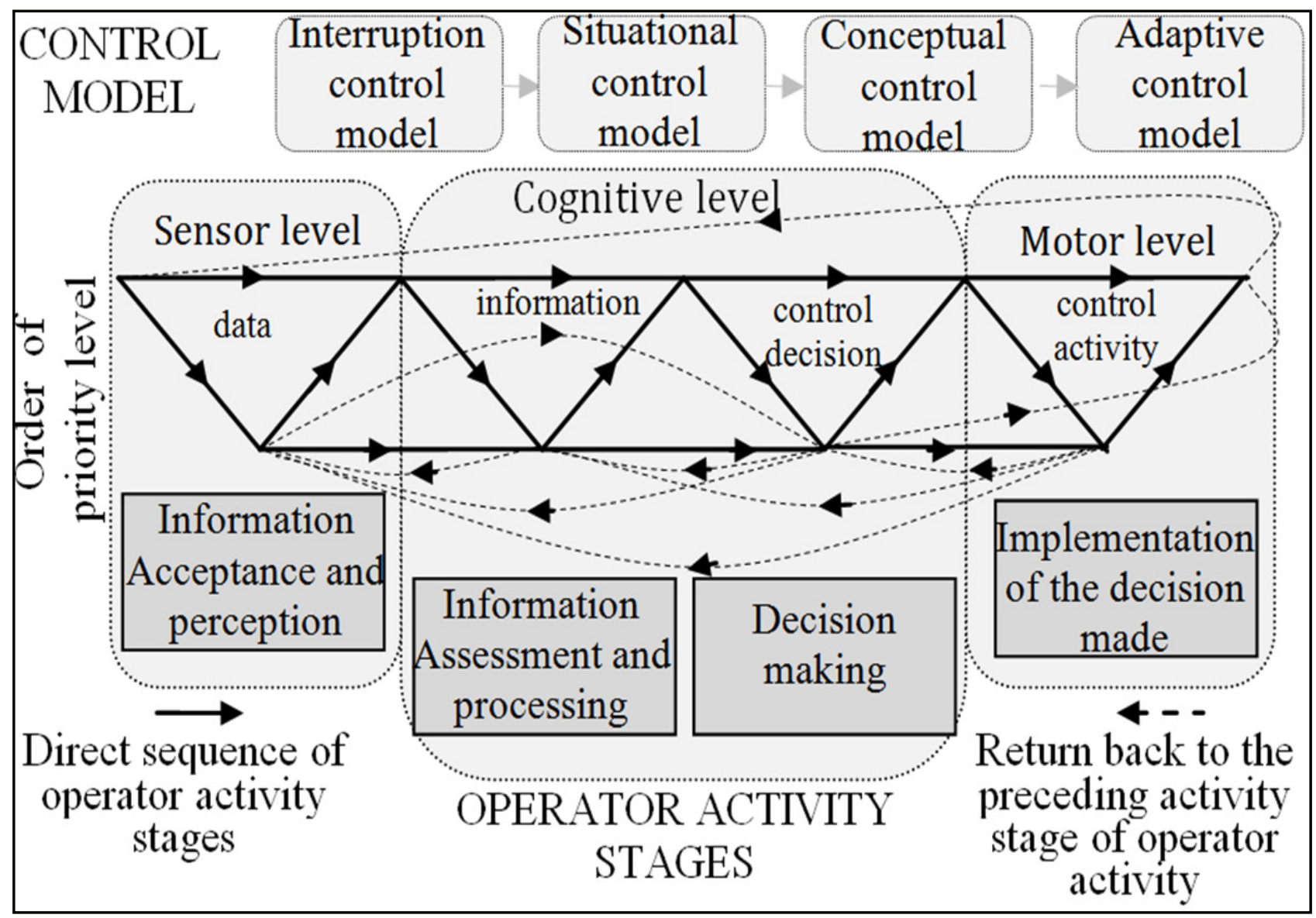

Figure 2. The system model of an operator's functions

At the present time one of the most widely accepted methods used for solving this task is an analytical laboratory experiment. According to it, in a laboratory environment a certain fragment of a professional activity is simulated while the rest are intentionally excepted, to a certain degree, it allows to segment separate stages of an operator's activity.

The test for a response to a mobile object (RMO) based on visual-motor monitoring (VMM) can be used here as a laboratory experiment for investigation of an operator's activity.

\section{MODEL OF VISUAL-MOTOR MONITORING}

According to the RMO test, the testee monitors movements of the object moving along the certain path and records the moment when the object position coincides with a test mark. The testee's task is to provide a maximum alignment of the moving object and the test mark by analyzing the speed and the path of the object movements and forecasting its position.

The physical model of the VMM task solving process in this case can be represented as an information feedback system (FB).

The FB coefficient - $k$ - determines the FB character and integrates objective information on results of performing operations and the operator's subjective information on the analysis data of his/her own actions.

If the operator has information on the results of his/her own actions ( $k \rightarrow 1$ ), according to the automatic control theory, it is possible to investigate the possibility of achieving a quality indicator of the system functioning, i.e. to investigate the testee's capability to analyze and correct his/her own actions.

In case of lack of information on the results of one's own actions $(k \rightarrow 0)$, the FB influence de- 
creases that allows to investigate the source system, i.e. the capability to the VMM.

The value of the $k$ coefficient is determined through the $k 1$ and $k 2$ coefficients as it is shown in Figure 3.

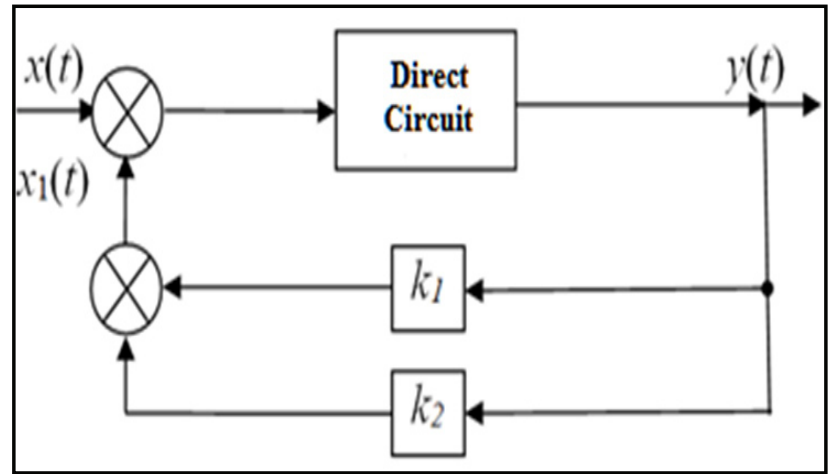

Figure 3. The physical model of the VMM task solving process

where $x(t)$ is an input value (information on the object monitored), $y(t)$ is an output value (the result of the operator's motor action), $x 1(t)$ is a FB effect (correction of the operator's actions basing on the current information on the results of control), $k 1$ is a FB coefficient, on the results of one's own activity analysis basing on the objective information, $k 2$ is a FB coefficient, on the results of one's own activity analysis basing on the subjective information.

These two FB loops represent the circuits for control on the bases of objective and subjective data.

Under conditions when the testee has an opportunity to analyze the objective information on the results of his/her own actions, $k 1>>k 2$.

While if the testee has no opportunity to analyze the objective information on the results of his/her own actions, then it may mean the decrease of the FB influence. At the same time signification of the $k 2$ coefficient increases. The full elimination of FB is impossible due to the operator's unconscious analysis of his/her own actions.

Therefore, it is evident that the feedback in the system will exist both in the presence of the objective information on the results of one's own activity and without it.

Now we can formalize this idea of the VMM process. In this case interaction of the operator and the technical system can be represented as a monitoring system working in deviation, where $\mathrm{x}(\mathrm{t})$ is a setting action, $\mathrm{v}(\mathrm{t})$ is a disturbance, $\mathrm{e}(\mathrm{t})$ is a error value, $\mathrm{CO}$ is a controlled object, Figure 4.

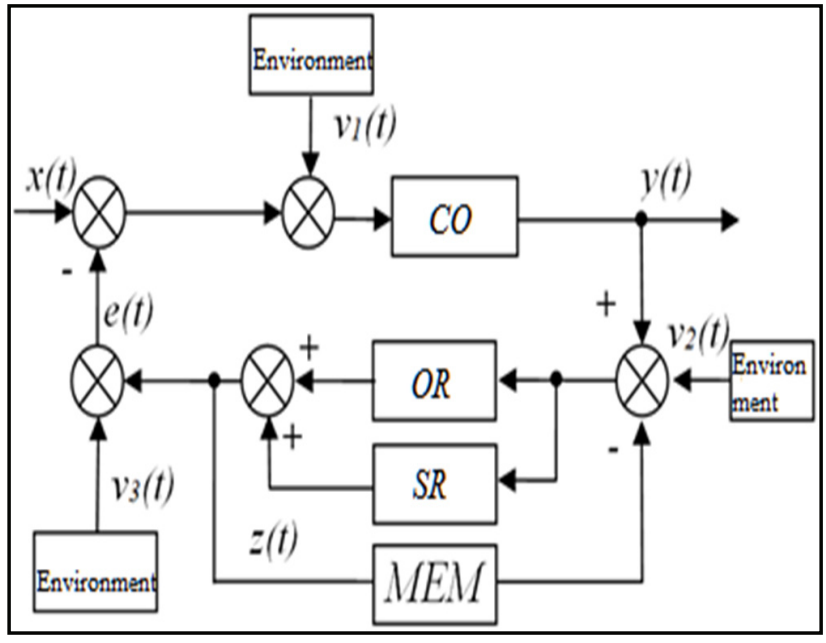

Figure 4. The model of interaction between the operator and the technical system in VMM

The operator's function is monitoring the system so that during the time the $y(t)$ output value would change in accordance with changing of the $x(t)$ setting action and little depend on $v(t)$, which is a combination of disturbances $v 1(t), v 2(t), v 3(t)$.

The feedback for deviation control is represented by two components: OR is a feedback for control on the basis of objective data, and SR is a feedback for control on the basis of subjective data. In addition to it, the operator's $z(t)$ feedback is introduced which realizes the function of the previous state memory (MEM). The memory function provides the analysis of result comparison of the $\mathrm{e}\left(t_{i-1}\right)$ deviation at the previous stage and the $e\left(t_{i}\right)$ deviation at the current stage taking into account the chosen control strategy for its correction at the next stage.

\section{RESULTS}

The hypothesis is formulated according to which the operator's actions in the VMM task solving process should be characterized by correlation. Furthermore, the data obtained under the OR conditions should have a greater statistical dependence in contrast to the data obtained under the SR conditions.

For the purpose of hypothesis checking the statistical analysis of the results of the VMM task solving investigation was performed. The VMM represents in a common case a random process of the $X(t)$ parameter measurement under conditions of the natural experiment.

The RMO test with the object of a steady speed 
der conditions of contradictory data from the OR and SR circuit is of an undoubted interest.

The research results were obtained by the grant support № 25.1095.2017/ПЧ.

\section{REFERENCES}

1) Festinger, L. A theory of cognitive dissonance. // Leon Festinger - Evanstone, IL: Row, Peterson, $1957-291$ p.

2) Metin I., The Advances in the History of Cognitive Dissonance Theory. // Irem Metin, Selin Metin Camgoz - International Journal of Humanities and Social Science, Vol. 1 No. 6, June 2011 - pp. 131-136.

3) Harmon-Jones E. Cognitive Dissonance Theory. //E. Harmon-Jones - V.S. Ramachandran (ed.) The Encyclopedia of Human Behavior, vol. 1 - Academic Press: 2012. - pp. 543-549.

4) Dickinson, D. Cognitive Dissonance, Pessimism, and Behavioral Spillover Effects // David L. Dickinson, Robert J. Oxoby - IZA Discussion Paper No. 2832 - 29 p.

5) Khodakov V.E. Methods for assessment of knowledge contradictory degree us- ing dissonance relation // V.E. Khodakov, V.G.Sherstjuk, K.G.Stepansky, A.A.Didyk, A.N.Martynov - Radio Electronics and Informatics. 1998, No.1 (2).

6) Petukhov I., Steshina L. Assessment of vocational aptitude of man-machine systems operators // Proceedings 7th International Conference on Human System Interactions (HSI), 16-18 June 2014, Costa da Caparica, Portugal, 2014, 44 - 48. 10.1109/HSI .2014.6860446

7) Beregovoy G.T. Psychological basis of human-operator training for activity under extreme conditions // G.T. Beregovoy, V.A.Ponomarenko - Psychology Issues. 1983, No. 1 - Pages 23-32. - https://www.voppsy.ru/contents/c831.htm

Paper sent to revision: 15.05.2017.

Paper ready for publication: 30.07.2017. 